\title{
Tunable room-temperature spin galvanic and spin Hall effects in van der Waals heterostructures
}

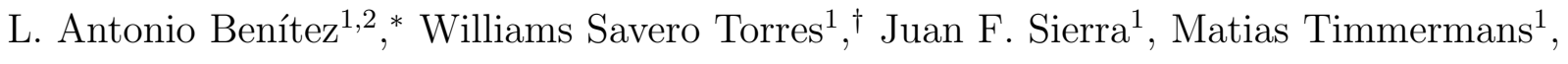 \\ Jose H. Garcia ${ }^{1}$, Stephan Roche ${ }^{1,3}$, Marius V. Costache ${ }^{1}$, and Sergio O. Valenzuela ${ }^{1,2}$ 拥 \\ ${ }^{1}$ Catalan Institute of Nanoscience and Nanotechnology (ICN2), \\ CSIC and The Barcelona Institute of Science and Technology (BIST), \\ Campus UAB, Bellaterra, 08193 Barcelona, Spain \\ ${ }^{2}$ Universitat Autònoma de Barcelona, \\ Bellaterra, 08193 Barcelona, Spain and \\ ${ }^{3}$ Institució Catalana de Recerca i Estudis Avançats (ICREA), 08010 Barcelona, Spain
}

(Dated: August 28, 2019)

\begin{abstract}
Spin-orbit coupling stands as a powerful tool to interconvert charge and spin currents and to manipulate the magnetization of magnetic materials through the spin torque phenomena [1-5]. However, despite the diversity of existing bulk materials and the recent advent of interfacial and low-dimensional effects [2, 3, 6], control of the interconvertion at room-temperature remains elusive. Here, we unequivocally demonstrate strongly enhanced room-temperature spin-to-charge (StC) conversion in graphene driven by the proximity of a semiconducting transition metal dichalcogenide $\left(\mathrm{WS}_{2}\right)$. By performing spin precession experiments in properly designed Hall bars, we separate the contributions of the spin Hall and the spin galvanic effects. Remarkably, their corresponding conversion efficiencies can be tailored by electrostatic gating in magnitude and sign, peaking nearby the charge neutrality point with a magnitude that is comparable to the largest efficiencies reported to date [6, 7]. Such an unprecedented electric-field tunability provides a new building block for spin generation free from magnetic materials and for ultra-compact magnetic memory technologies.
\end{abstract}

\footnotetext{
* These authors contributed equally to this work; an.benitez7@gmail.com

$\dagger$ These authors contributed equally to this work; williams.savero@icn2.cat

$\ddagger$ SOV@icrea.cat
} 
While classical spintronics relies on the generation and manipulation of spin-polarized electrical currents using ferromagnetic materials, the emerging field of spin orbitronics is driven by the creation of pure spin currents in non-magnetic materials by means of the spinorbit interaction (SOI) [1-4]. In the spin Hall effect (SHE) and the inverse spin galvanic effect (ISGE), a charge current generates a transverse spin current and a non-equilibrium spin density, respectively, that can be utilized, for example, to manipulate the magnetization of ferromagnets in non-volatile memory technologies [8]. Various categories of materials are being intensively investigated for such a purpose, including metals, oxides and topological insulators [1 [5, 9]. Two-dimensional (2D) atomic crystals constitute a unique platform to engineer materials with novel functionalities in the limit of ultra-compact device architectures [10, 11]. Because they consist of atomically thin planes, their electrical, optical and spin properties can be enriched and tailored by proximity effects. In recent years, magnetic correlations and strong SOI have been successfully imprinted onto graphene and observed at room temperature [12 14]. When graphene is in direct proximity to a transition metal dichalcogenide (TMDC), it preserves its electronic properties while acquiring a complex spin texture with out-of-plane and winding in-plane components (Fig. 1a) that result in anisotropic spin dynamics [14-18]. Theoretical studies further suggest that the unique nature of this SOI can enhance the graphene StC conversion efficiency [19, 20], while the coexistence of SHE and IGSE is allowed by new SOI terms such as the valley Zeeman coupling [15, 16. The observation of the SHE was originally reported in graphene decorated with adatoms and in contact with $\mathrm{WS}_{2}$ [21] but follow-up studies suggest that the detected signal was not spin related [22 24]. More recently, the SHE was observed in heterostructures comprising multilayer graphene and $\mathrm{MoS}_{2}$ [25]. However, because $\mathrm{MoS}_{2}$ is believed to be conductive and the experiment does not allow control of the carrier density of neither graphene nor $\mathrm{MoS}_{2}$, it is not possible to fully discriminate between $\mathrm{StC}$ conversion by proximity effects and in the bulk of $\mathrm{MoS}_{2}[25]$.

In this work, we demonstrate large, gate-tunable StC conversion driven by both the SHE and ISGE in monolayer graphene- $\mathrm{WS}_{2}$ heterostructures at room temperature. The SHE and ISGE are found to coexist in a narrow energy region nearby the charge neutrality point (CNP), enabling the control of both phenomena by tuning the carrier density $n$. The StC conversion mechanisms manifest in the presence of large spin transport anisotropy, as a consequence of the inherited spin textures of the graphene states. The observed SHE 
conversion efficiency as a function of $n$ and temperature is well reproduced by theoretical calculations of the spin Hall conductivity.

The measurement scheme and optical image of a typical device are shown in Figs. 1b and 1c, respectively [26 28] (see Methods for fabrication details and Supplementary Fig. 1 for detailed device schematics). A carefully designed experimental protocol based on spin precession [29] allows us to isolate the proximity-induced StC conversion in the modified graphene (graphene- $\mathrm{WS}_{2}$ ) from both spurious phenomena and competing StC conversion occurring in the bulk of the TMDC [25]. The device consists of a patterned graphene Hall cross with a $\mathrm{WS}_{2}$ flake along one of the arms and ferromagnetic injector/detector electrodes (F1, F2, F3) across the other. An electric field $E$ along the graphene- $\mathrm{WS}_{2}$ arm (and associated current I, Fig. 1b) generates a spin current and spin accumulation due to the SHE (red arrows) and a non-equilibrium spin density conveyed by the ISGE (blue arrow, see Fig. 1a). The spins, carrying information on the SHE and the ISGE, diffuse in the graphene and are detected by measuring the non-local voltage $V_{\mathrm{nl}}^{\mathrm{F}}$ at F1. Alternatively, a spin current in graphene can be generated by applying $I$ in F1. In this case, the spin current and non-equilibrium spin density that reach the graphene(gr)- $\mathrm{WS}_{2}$ are converted into a voltage $V_{\mathrm{nl}}^{\mathrm{gr}-\mathrm{WS}_{2}}$ by the reciprocal effects: the inverse spin Hall effect (ISHE) and the spin galvanic effect (SGE). In the linear regime, for phenomena that are Onsager reciprocals such as the SHE and the ISHE, $V_{\mathrm{nl}}^{\mathrm{F}}=V_{\mathrm{nl}}^{\mathrm{gr}-\mathrm{WS}_{2}}=V_{\mathrm{nl}}$.

For the StC conversion experiments, F1 and F2 are equivalent and either of them can be used to investigate the SHE, the ISGE and their reciprocals. In addition, F2 and F3 (in combination with F1) are used to independently characterize the spin dynamics in both the pristine graphene and the graphene- $\mathrm{WS}_{2}$ [14] (Supplementary Section I). This allows us to determine the StC conversion efficiencies without extra fitting parameters. The out-of-plane and in-plane spin relaxation lengths in graphene- $\mathrm{WS}_{2}, \lambda_{\mathrm{s}}^{\perp}$ and $\lambda_{\mathrm{s}}^{\|}$, are about one-micrometre and few-hundred nanometres, respectively [14]. Thus, the width $l_{\mathrm{w}}$ of the $\mathrm{WS}_{2}$ flake is selected to be $l_{\mathrm{w}} \sim 1 \mu \mathrm{m}$ to simultaneously detect the SHE and the ISGE [1]. Sufficiently large distances from the $\mathrm{WS}_{2}$ to $\mathrm{F} 1$ and F2 ensure large-angle spin precession at low magnetic fields, for which the magnetization of the electrodes $\mathbf{M}_{1,2}$ remain unaffected [30]. A backgate voltage $V_{\mathrm{g}}$ is applied to the substrate to tune the carrier density of both the graphene and $\mathrm{WS}_{2}$.

For graphene-WS $\mathrm{W}_{2}$ in the $x y$-plane and $I$ applied along $\hat{\mathbf{y}}$ (Fig. 1b), the spins generated 
by the SHE are polarized in the $\hat{\mathbf{z}}$ direction while those generated by the ISGE are in the $\hat{\mathbf{x}}$ direction (see Fig. 1a). Because the ISGE and SHE spins are orthogonal, their contributions to $V_{\mathrm{nl}}$ can be readily separated using spin precession [29]. $V_{\mathrm{nl}}$ is proportional to the local spin accumulation projected along the detector magnetization $\mathbf{M}_{1}$. Due to magnetic shape anisotropy, $\mathbf{M}_{1}$ tends to be aligned with the F1 length, which is set along $\hat{\mathbf{y}}$, orthogonal to the spins generated by both the SHE and the ISGE (Fig. 1b). Thus, at zero magnetic field, $V_{\mathrm{nl}}=0$ since neither the SHE nor the ISGE contribute to $V_{\mathrm{nl}}$. For a low magnetic field $B_{z}$ perpendicular to the graphene plane, $V_{\mathrm{nl}}$ solely depends on the ISGE. While the SHE spins are parallel to $B_{z}$ and remain unaffected, the ISGE spins undergo precession. Because the ISGE spins are no longer perpendicular to $\mathbf{M}_{1}$ when they reach $\mathrm{F} 1, V_{\mathrm{nl}} \neq 0$ (Fig. 1d). In contrast, for an in-plane magnetic field $B_{x}$, the ISGE spins remain unaffected so that $V_{\mathrm{nl}}$ is solely dependent on the SHE (Fig. 1e). Similar arguments can be applied to the ISHE and the SGE.

For the transport to occur only in the modified graphene, it is crucial to verify the insulating character of $\mathrm{WS}_{2}$. Otherwise, a current in $\mathrm{WS}_{2}$ could generate both out-of-plane and in-plane spins, e.g. through the SHE in $\mathrm{WS}_{2}$ [25], which can be mistaken with spins associated to the proximity effects. Figure 2 a shows the current $I_{\mathrm{ds}}$ versus $V_{\mathrm{g}}$ when a constant driving voltage $V_{\mathrm{ds}}$ is applied between graphene and $\mathrm{WS}_{2} . I_{\mathrm{ds}}$ increases sharply at $V_{\mathrm{g}}^{\mathrm{T}} \sim 12$ $\mathrm{V}$, marking the $V_{\mathrm{g}}$ at which $\mathrm{WS}_{2}$ becomes conducting. Therefore, to avoid spin absorption (spin transfer) to (from) $\mathrm{WS}_{2}$, all $\mathrm{StC}$ conversion measurements are acquired at $V_{\mathrm{g}}<V_{\mathrm{g}}^{\mathrm{T}}$. Figure $2 \mathrm{~b}$ shows the gate-dependent resistance $R$ of the graphene- $\mathrm{WS}_{2}$. In this region, the graphene is $n$-doped with the CNP at $V_{\mathrm{g}}^{\mathrm{CNP}}=-10 \mathrm{~V}$. The fact that $V_{\mathrm{g}}^{\mathrm{CNP}}<V_{\mathrm{g}}^{\mathrm{T}}$ allows us to study the characteristic StC conversion for both electrons and holes.

The insets of Figs. 2c and 2d show typical $R_{\mathrm{nl}}^{\downarrow, \uparrow}=V_{\mathrm{nl}}^{\downarrow \uparrow \uparrow} / I$ versus applied magnetic field with $\mathbf{M}_{1}$ antiparallel $\left(V_{\mathrm{nl}}^{\downarrow}\right)$ and parallel $\left(V_{\mathrm{nl}}^{\uparrow}\right)$ to $\hat{\mathbf{y}}$ (see Fig. 1b). The current $I$ is applied at $\mathrm{F} 1$ and $V_{\mathrm{nl}}^{\downarrow \uparrow \uparrow}$ measured in the graphene across the graphene- $\mathrm{WS}_{2}$ arm. The orientation of $\mathbf{M}_{1}$ is prepared prior to the measurements by applying a magnetic field along $\hat{\mathbf{y}}$ with a magnitude exceeding the F1 coercive field. The inversion of the magnetic-field dependence when $\mathbf{M}_{1}$ reverses demonstrates the spin-related origin of the signal. The main panels of Figs. 2c and 2d show $\Delta R_{\mathrm{nl}} \equiv R_{\mathrm{nl}}^{\uparrow}-R_{\mathrm{nl}}^{\downarrow}$. By calculating the difference between $R_{\mathrm{nl}}^{\uparrow}$ and $R_{\mathrm{nl}}^{\downarrow}$, any non-spin related component in $V_{\mathrm{n} l}^{\downarrow, \uparrow}$ is eliminated.

In Fig. 2c, the magnetic field is applied in-plane in the ISHE configuration, as repre- 
sented in Fig. 1e; therefore $\Delta R_{\mathrm{nl}}$ corresponds to the ISHE signal $R_{\mathrm{ISHE}}$. In Fig. $2 \mathrm{~d}$, the magnetic field is applied out-of-plane in the SGE configuration, as represented in Fig. 1d, and thus $\Delta R_{\mathrm{nl}} \equiv R_{\mathrm{SGE}}$. The experimental data in Figs. 2c and 2d exhibit an antisymmetric spin precession lineshape [29]. The spins injected by F1 are parallel to $\hat{\mathbf{y}}$ and, consequently, only when the magnetic field generates a $\hat{\mathbf{z}}(\hat{\mathbf{x}})$ spin component, a nonzero $R_{\mathrm{ISHE}}\left(R_{\mathrm{SGE}}\right)$ is detected. At low magnetic fields $R_{\mathrm{ISHE}}$ and $R_{\mathrm{SGE}}$ are approximately linear; they reach maximum magnitude at about $\pm 50 \mathrm{mT}$ and then decrease. The extrema in $R_{\mathrm{ISHE}}$ and $R_{\mathrm{SGE}}$ indicate an aggregate spin precession angle of $\pi / 2$ when reaching the Hall cross. The asymptotic decrease to zero at larger magnetic fields $(>50 \mathrm{mT})$ is associated to spin dephasing.

The StC conversion efficiencies can be obtained from the spin precession lineshapes in Figs. 2c and 2d. To reduce the number of unknown parameters, the spin dynamics outside and inside the graphene- $\mathrm{WS}_{2}$ region is independently characterized as follows. Figure 2e shows typical non-local spin precession measurements $r_{\mathrm{nl}}=V_{\mathrm{nl}}^{\downarrow \uparrow \uparrow \uparrow} / I$ in pristine graphene using electrodes F1 and F3. Measurements are performed for antiparallel $(\downarrow \uparrow)$ and parallel $(\uparrow \uparrow)$ configurations of $\mathbf{M}_{1,3}$ and magnetic fields $B_{z}$ and $B_{x}$ (see Supplementary Fig. 2 for $B_{z}$ measurements). By fitting the results to the solution of the Bloch equations, the spin transport properties of the pristine graphene are extracted (Supplementary Fig. 2). Figure $2 \mathrm{f}$ shows the spin precession response in graphene- $\mathrm{WS}_{2}$ as a function of $B_{x}$ using electrodes F1 and F2. The results highlight the strongly anisotropic character of the spin transport, in agreement with prior reports [14]. In particular, they show that as $B_{x}$ increases, the corresponding $r_{\mathrm{nl}}$ becomes much larger than its value at $B=0$, which demonstrates that that the spin lifetime of spins in the plane of graphene- $\mathrm{WS}_{2}, \tau_{\mathrm{s}}^{\|}$, is much smaller than the lifetime of spins pointing out of plane, $\tau_{\mathrm{s}}^{\perp}$. Their magnitudes are calculated by modelling the spin diffusion in the graphene and the graphene- $\mathrm{WS}_{2}$ with the parameters extracted from Fig. 2e, yielding $\tau_{\mathrm{s}}^{\|}=(6 \pm 1)$ ps and $\tau_{\mathrm{s}}^{\perp}=(52 \pm 10)$ ps. The anisotropy ratio, defined as $\zeta=\tau_{\mathrm{s}}^{\perp} / \tau_{\mathrm{s}}^{\|}$is $\zeta=8 \pm 3$ (Supplementary Section II). With the spin dynamics fully characterized, the spin and spin-current densities can be calculated, at any position, from the analytical solution of the Bloch diffusion equation (Supplementary Section II).

At this point, the spin precession lineshapes in Figs. 2c and 2d can be readily obtained from the spin current density $j_{\mathrm{s}}^{z}$ associated to the spins projected in $\hat{\mathbf{z}}$ (ISHE) and the inplane component of the spin density in $\hat{\mathbf{x}}(\mathrm{SGE})$ in the graphene- $\mathrm{WS}_{2}$ region (Fig. 1b). The only unknown parameters are the corresponding $\mathrm{StC}$ conversion efficiencies, which manifest 
as scaling factors (Supplementary Section II). In the ISHE case, the efficiency is quantified with the spin Hall angle $\theta_{\mathrm{SHE}}$, which measures the conversion from $j_{\mathrm{s}}^{z}$ to a charge current density $j_{\mathrm{c}}^{y}[1]$. In the case of the $\mathrm{SGE}$, where a spin density is converted into $j_{\mathrm{c}}^{y}$, several figures of merit have been proposed. In our experiments, the spin density leading to $R_{\mathrm{SGE}}$ results directly from the spin current density $j_{\mathrm{s}}^{x}$. Therefore, it is possible to adopt an SGE equivalent to $\theta_{\mathrm{ISHE}}$, namely $\alpha_{\mathrm{SGE}} \equiv j_{\mathrm{c}}^{y} / j_{\mathrm{s}}^{x}$. Because the latter conversion is from a $2 \mathrm{D}$ spin current into a $2 \mathrm{D}$ charge current, $\alpha_{\mathrm{SGE}}$ is dimensionless. This contrasts with the commonly used inverse Edelstein effect length $\lambda_{\text {IEE }}$, which quantifies the SGE conversion efficiency from a three-dimensional spin current into a 2D charge current and has the dimension of a length [6].

The solid lines in Figs. 2c and 2d represent the calculated responses from which roomtemperature $\theta_{\mathrm{SHE}} \approx 0.3 \%$, and $\alpha_{\mathrm{SGE}} \approx 0.1 \%$ are estimated. The agreement between the model and experiment is excellent considering that only one adjustable (scaling) parameter is used. For comparison purposes, one can convert $\alpha_{\mathrm{SGE}}$ and $\theta_{\mathrm{SHE}}$ into $\lambda_{\mathrm{IEE}}$ through $\lambda_{\mathrm{IEE}}^{\mathrm{SGE}}=$ $\alpha_{\mathrm{SGE}} \lambda_{\mathrm{s}}^{\|} \approx 0.42 \mathrm{~nm}$ and $\lambda_{\mathrm{IEE}}^{\mathrm{SHE}}=\theta_{\mathrm{SHE}} \lambda_{\mathrm{s}}^{\perp} \approx 3.75 \mathrm{~nm}$, where $\lambda_{\mathrm{s}}^{\|}=420 \mathrm{~nm}$ and $\lambda_{\mathrm{s}}^{\perp}=1.25$ $\mu \mathrm{m}$ are the in-plane and out-of-plane spin relaxation lengths in graphene- $\mathrm{WS}_{2}$. Remarkably, these values compare very favourably with those estimated for heavy metals as $\theta_{\mathrm{SHE}} \lambda_{\mathrm{s}}^{\mathrm{M}}\left(\lambda_{\mathrm{s}}^{\mathrm{M}}\right.$ the metal spin diffusion length), yielding $0.2 \mathrm{~nm}$ for Pt, $0.3 \mathrm{~nm}$ for Ta and 0.43 for $\mathrm{W}$ [31. Furthermore, $\lambda_{\mathrm{IEE}}^{\mathrm{SHE}}$ for graphene- $\mathrm{WS}_{2}$ is larger than the $\lambda_{\mathrm{IEE}}$ reported for any device at room temperature, being one order of magnitude larger than in $\mathrm{Bi} / \mathrm{Ag}$ interfaces, $\lambda_{\mathrm{IEE}}=0.2-0.33$ $\mathrm{nm}$ [6], and almost twice $\lambda_{\text {IEE }}=2.1 \mathrm{~nm}$ in the topological insulator $\alpha$-Sn [7].

The ISGE and SGE can also be investigated when $\mathbf{M}_{1}$ rotates and develops a component along $\hat{\mathbf{x}}$ with sufficiently large $B_{x}[26$, 28]. As observed in Fig. 2e, the magnetization rotation is evident for $B_{x}>0.2 \mathrm{~T}$, with $\mathbf{M}_{1}$ (and $\mathbf{M}_{3}$ ) becoming fully aligned with $B_{x}$ for $\left|B_{x}\right|>0.3 \mathrm{~T}$. Therefore, a broad step in $R_{\mathrm{nl}}$ is expected for $B_{x}$ between -0.3 and 0.3 T. Figure 3a shows $R_{\mathrm{nl}}$ versus $B_{x}$ for three representative gate voltages: at the CNP $\left(V_{\mathrm{g}}=-10 \mathrm{~V}\right)$ and for electron $\left(V_{\mathrm{g}}=-3 \mathrm{~V}\right)$ and hole $\left(V_{\mathrm{g}}=-13 \mathrm{~V}\right)$ conduction. The SGE and the ISHE signals are clearly observed. The solid lines represent $M_{1 x}$, the projected $\mathbf{M}_{1}$ along $\hat{\mathbf{x}}$, which is independently extracted using measurements as in Fig. 2e [26]. The spin precession response associated to the ISHE, as shown in Fig. 2c, appears superimposed. The agreement between the fielddependence of $M_{1 x}$ and the SGE step is excellent. In addition, the magnitude of the SGE extracted from these measurements coincides with the results obtained from spin precession 
(Fig. 2d); this allows us to discard any artifacts associated with stray magnetic fields.

The results in Fig. 3a suggest that the ISHE and the SGE follow distinct carrier density dependences. While the SHE signal is clearly distinguished at the CNP, the SGE signal changes sign between electrons and holes and becomes undetectable at the CNP. Figure $3 \mathrm{~b}$ represents the room-temperature magnitude of the ISHE and SGE versus $V_{\mathrm{g}}$. The SGE is quantified by means of the step height, $R_{\mathrm{SGE}}^{*} \equiv R_{\mathrm{nl}}(0.4 \mathrm{~T})-R_{\mathrm{nl}}(-0.4 \mathrm{~T})$ and the ISHE by the substraction $R_{\mathrm{ISHE}}^{*}$ between the values of $R_{\mathrm{ISHE}}$ at its two extrema (see Fig. 3a). It is observed that $n$ has a dramatic influence in the StC conversion efficiency. $R_{\mathrm{ISHE}}^{*}$ displays a sharp peak with its maximum located nearby the CNP; $R$ vs $V_{\mathrm{g}}$ is shown in Fig. $3 \mathrm{~b}$ for comparison (solid black line). In contrast, $R_{\mathrm{SGE}}^{*}$ is asymmetric about the CNP, changing sign between electrons and holes, as concluded from Fig. 3a.

The change in sign in $R_{\mathrm{SGE}}^{*}$ stems from the change in the nature of the carriers, as the winding of the spin texture is symmetrical about the CNP [16, 20]. This further confirms our interpretation of the signal as originating from the SGE. Theoretical calculations for graphene- $\mathrm{WS}_{2}$ suggest that $R_{\mathrm{ISHE}}$ also changes sign nearby the CNP with a behaviour qualitatively similar to that of $R_{\mathrm{SGE}}$. However, the magnitude of $R_{\mathrm{ISHE}}$ is expected to be much larger for holes than for electrons [19]. As a consequence, the smearing effect resulting from the presence of charge puddles and room-temperature broadening might suppress the extremum in the electron side, leaving only the dominant hole peak by the CNP. The results in Fig. 4a, showing $R_{\mathrm{ISHE}}^{*}$ versus $n$ as the temperature $T$ decreases, indicate that this hypothesis is plausible. Indeed, $R_{\mathrm{ISHE}}^{*}$ at room temperature $(300 \mathrm{~K})$ presents an incipient electron-hole asymmetry, with a steeper decrease for electron conduction. At $200 \mathrm{~K}$, the decrease becomes steeper as thermal broadening is reduced. At $100 \mathrm{~K}$ a negative minimum at $n=5 \times 10^{11}$ $\mathrm{cm}^{-2}$ becomes fully developed. Figure $4 \mathrm{~b}$ shows the computed spin Hall conductivity $\sigma_{x y}^{\mathrm{SHE}}$ in the weak disorder limit [19] (see Supplementary Section III). The parameters used for the calculations to match the experimental trends are within a factor two of those obtained by density functional theory [16, 19]. Notably, the theoretical model describes qualitatively all of the experimentally observed features, including the relative magnitude of the positive and negative extrema, their approximate width and the temperature $T \sim 200 \mathrm{~K}$ at which the change in sign is observed. Although the temperature dependence of $R_{\mathrm{ISHE}}^{*}$ is highly non-trivial, $R_{\mathrm{ISHE}}^{*}$ varies roughly as $1 / T$ nearby the extrema (Inset Fig. 4a), which is also well reproduced by the calculations (Inset Fig. 4b). The tendency for saturation in $R_{\mathrm{ISHE}}^{*}$ at 
low $T$ for hole conduction, which is not observed in the model, could indicate the presence of charge puddles or other types of impurities and defects.

The observation of the SGE and ISHE within the gap of $\mathrm{WS}_{2}$, their dependence on $n$ and the excellent agreement with theoretical expectations prove that the phenomena originate from SOI proximity effects in graphene. However, the magnitude of the SGE and ISHE and the large spin relaxation anisotropy suggest that the theoretical understanding is still incomplete and deserves further investigation. Theoretical results, which consider only intrinsic effects [19, 20], are compatible with the experimental $\theta_{\text {SHE }}$ only for weak intervalley scattering while yielding an $\alpha_{\mathrm{SGE}}$ that is at least one order of magnitude smaller than the experimental value. Furthermore, a large SHE, as observed in Fig. 2c, has been deemed incompatible with anisotropic spin relaxation (Fig. 2f). Anisotropic spin relaxation signals the presence of intervalley scattering, which effectively reduces the mass of the carriers and leads to the suppression of the SHE [17, 19]. Such discrepancies between experimental results and purely intrinsic effects suggest additional extrinsic contributions in the observed SHE and ISGE. A larger localized SOI can be mediated by localized defects, such as sulphur vacancies in $\mathrm{WS}_{2}$, while an effective enhancement of $\theta_{\mathrm{SHE}}$ could arise from resonant scattering as proposed for metallic adatoms [24, 32].

Our experiments therefore provide valuable insights into the physics underpinning proximity effects in graphene. In addition, they offer novel strategies to manipulate spin information in ultra-compact van der Waals heterostructures, which could further impact the design and performance of advanced magnetic memory technologies [8]. Indeed, in recent years the SHE and ISGE have evolved from subtle academic phenomena to effective approaches to electrically manipulate the magnetization of a ferromagnet [1, 8]. The electric-field control of the StC conversion, both in magnitude and sign, and the unprecedented efficiency thus open the door to magnetization control with 2D materials and to novel spin-logic circuits without the use of ferromagnets.

Note: After completion of the current research, a work studying the spin galvanic effect using magnetization rotation in graphene- $\mathrm{WS}_{2}$ has been reported [33]. The investigation presents results showing SGE modulation at $4.2 \mathrm{~K}$ and large $n$ and shows that the SGE signal persists up to room temperature. However, the SGE modulation and the SHE are not observed at room temperature. 


\section{METHODS}

Device Fabrication. van der Waals graphene- $\mathrm{WS}_{2}$ heterostructures were fabricated by dry viscoelastic stamping [14. The transfer set-up comprises an optical microscope with large working distance optical objectives (Nikon Eclipse Eclipse LV 100ND) and a three-axis micrometer stage. Graphene is obtained by mechanical exfoliating highly-oriented pyrolytic graphite (SPI Supplies) onto a $p$-doped $\mathrm{Si} / \mathrm{SiO}_{2}$ substrate. Large-area monolayer graphene is selected by optical contrast after a previous calibration with Raman spectroscopy. To fabricate the van der Waals heterostructure, $\mathrm{WS}_{2}$ flakes are transferred onto a viscoelastic stamp (Gelpack), which is then transferred on top of the graphene target. After assembling, the stacks are annealed for 1 hour at $500{ }^{\circ} \mathrm{C}$ in high vacuum $\left(10^{-8}\right.$ Torr $)$. The heterostructure is coated with a PMMA resin mask and patterned into a Hall cross bar using electron-beam lithography followed by oxygen plasma etching [34. The resin is then removed with acetone. The contact electrodes are defined in two electron-beam lithography steps, one for the normal metal electrodes, $\operatorname{Ti}(1 \mathrm{~nm})-\mathrm{Pd}(50 \mathrm{~nm})$ and the other for the ferromagnets, $\mathrm{TiO}_{\mathrm{x}} / \mathrm{Co}(30 \mathrm{~nm})$. The contact materials are deposited by electron-beam evaporation in a chamber with base pressure of $10^{-8}$ Torr. The $\mathrm{TiO}_{\mathrm{x}}$ barriers are fabricated by evaporating $4 \AA+4 \AA$ of $\mathrm{Ti}$ and 30 min oxidation after each evaporation in an oxygen atmosphere of about $10^{-1}$ Torr.

Electrical characterization. The devices are wired to a chip carrier that is placed in a cryogen-free cryostat. Charge transport properties were characterized by means of two- and fourterminal measurements. The contact resistance in the $\mathrm{TiO}_{\mathrm{x}} / \mathrm{Co}$ electrodes are larger than $10 \mathrm{k} \Omega$. The typical average electron/hole mobility is in the range of $\mu=5000 \mathrm{~cm}^{2} \mathrm{~V}^{-1} \mathrm{~s}^{-1}$ with a residual carrier density of $2.5 \times 10^{11} \mathrm{~cm}^{-2}$. A back-gate voltage applied to the $p$-doped Si subtrate is used to control the carrier density $n$ in the device.

Data Availability. The data that support the plots within this paper and other findings of this study are available from the corresponding authors upon reasonable request.

[1] Sinova, J., Valenzuela, S. O., Wunderlich, J., Back, C. H., \& Jungwirth, T. Spin Hall effects. Rev. Mod. Phys. 87, 1213 (2015).

[2] Manchon A., Koo, H. C., Nitta, J., Frolov, S. M., \& Duine, R. A. New perspectives for Rashba spin-orbit coupling. Nature Mater. 14, 871-882 (2015). 
[3] Soumyanarayanan, A., Reyren, N., Fert. A. \& Panagopoulos, C. Emergent phenomena induced by spin-orbit coupling at surfaces and interfaces. Nature 539, 509-517 (2016).

[4] Sander, D. et al. The 2017 magnetism roadmap. J. Phys. D: Appl. Phys. 50, 363001 (2017).

[5] Manchon A., Železný, J., Miron, I. M., Jungwirth, T., Sinova, J., Thiaville, A., Garello, K., \& Gambardella, P. Current-induced spin-orbit torques in ferromagnetic and antiferromagnetic systems. Rev. Mod. Phys. accepted (2019).

[6] Rojas Sánchez, J. C., Vila, L., Desfonds, G., Gambarelli, S., Attan, J. P., De Teresa, J. M., Magn, C. \& Fert, A. Spin-to-charge conversion using Rashba coupling at the interface between non-magnetic materials. Nat. Commun. 4, 1944 (2013).

[7] Rojas-Sánchez, J.-C., Oyarzún, S., Fu, Y., Marty, A., Vergnaud, C., Gambarelli, S., Vila, L., Jamet, M., Ohtsubo, Y., Taleb-Ibrahimi, A., Le Fèvre, P., Bertran, F., Reyren, N., George, J.-M., \& Fert, A. Spin to Charge Conversion at Room Temperature by Spin Pumping into a New Type of Topological Insulator: $\alpha$-Sn Films. Phys. Rev. Lett. 116, 096602 (2016).

[8] Garello, K., Yasin, F., Couet, S., Souriau, L., Swerts, J., Rao, S., Van Beek, S., Kim, W., Liu, E., Kundu, S., Tsvetanova, D., Jossart, N., Croes, K., Grimaldi, E., Baumgartner, M., Crotti, D., Furnémont, A., Gambardella, P. \& Kar, G. S. SOT-MRAM 300MM Integration for Low Power and Ultrafast Embedded Memories. 2018 IEEE Symposium on VLSI Circuits Digest of Technical Papers 81-82 (2018).

[9] Bibes, M. \& Barthélémy, A. Oxide spintronics. IEEE Trans. Electron. Devices 54, 1003-1023 (2007).

[10] Geim, A. K. \& Grigorieva, I. V. Van der Waals heterostructures. Nature 499, 419-425 (2013).

[11] Novoselov, K. S., Mishchenko, A., Carvalho, A. \& Castro Neto, A. H. 2D materials and van der Waals heterostructures. Science 353, aac9439 (2016).

[12] Wang, Z., Tang, C., Sachs, R., Barlas, Y., \& Shi, J. Proximity-Induced Ferromagnetism in Graphene Revealed by the Anomalous Hall Effect on transition-metal dichalcogenides. Phys. Rev. Lett. 114, 016603 (2015).

[13] Leutenantsmeyer, J. C., Kaverzin, A. A., Wojtaszek, M. \& van Wees, B.J. Proximity induced room temperature ferromagnetism in graphene probed with spin currents. 2D Mater. 4, 014001 (2017).

[14] Benítez, L. A., Sierra, J. F., Savero Torres, W., Arrighi, A., Bonell, F., Costache, M. V. \& Valenzuela, S. O. Strongly anisotropic spin relaxation in graphene-transition metal dichalco- 
genide heterostructures at room temperature. Nat. Phys. 14, 303-308 (2018).

[15] Gmitra, M. \& Fabian, J. Graphene on transition-metal dichalcogenides: A platform for proximity spin-orbit physics and optospintronics. Phys. Rev. B 92, 155403 (2015).

[16] Gmitra, M., Kochan, D., Högl, P. \& Fabian, J. Trivial and inverted Dirac bands and the emergence of quantum spin Hall states in graphene on transition-metal dichalcogenides. Phys. Rev. B 93, 155104 (2016).

[17] Cummings, A. W., Garcia, J. H., Fabian, J.,\& Roche, S. Giant Spin Lifetime Anisotropy in Graphene Induced by Proximity Effects. Phys. Rev. Lett. 119, 206601 (2017).

[18] Ghiasi, T. S., Ingla-Ayns, J., Kaverzin, A. A., \& van Wees, B. J. Large Proximity-induced spin lifetime anisotropy in transition-metal dichalcogenide/graphene heterostructures Nano Lett. 17, 7528-7532 (2017).

[19] Garcia, J. H., Cummings, A.W., \& Roche, S. Spin Hall effect and weak antilocalization in graphene/transition metal dichalcogenide heterostructures. Nano Lett. 17, 5078 (2017).

[20] Offidani, M., Milletarì, M., Raimondi, R., \& Ferreira, A. Optimal charge-to-spin conversion in graphene on transition metal dichalcogenide. Phys. Rev. Lett. 119, 0196801 (2017).

[21] Avsar, A., Tan, J. Y., Taychatanapat, T., Balakrishnan,J., Koon, G.K.W., Yeo, Y., Lahiri, J., Carvalho, A., Rodin, A. S., O’Farrell, E.C.T., Eda, G., Castro Neto, A. H. \& Özyilmaz, B. Spin-orbit proximity effect in graphene. Nat. Commun. 5, 4875 (2014).

[22] Kaverzin, A. A. \& van Wees, B. J. Electron transport nonlocality in monolayer graphene modified with hydrogen silsesquioxane polymerization Phys. Rev. B 91, 165412 (2015).

[23] Wang, Y., Cai, X., Reutt-Robey, J., \& Fuhrer, M. S. Neutral-current Hall effects in disordered graphene. Phys. Rev. B 92, 161411(R) (2015).

[24] Van Tuan, D., Marmoleho-Tejada, J. M., Waintail, X., Nikolić, B. K., Valenzuela, S. O. \& Roche, S. Spin Hall Effect and Origins of Nonlocal Resistance in Adatom-Decorated Graphene. Phys. Rev. Lett. 117, 176602 (2016).

[25] Safeer, C. K., Ingla-Aynés, J., Herling, F., Garcia, J. H., Vila, M., Ontoso, N., Calvo, M. R., Roche, S., Hueso, L. E. \& Casanova, F. Room-Temperature Spin Hall Effect in Graphene/MoS 2 van der Waals Heterostructures Nano Lett. 19, 1074-1082 (2019).

[26] Valenzuela, S. O.. \& Tinkham, M. Direct electronic measurement of the spin Hall effect. Nature 442, 176-179 (2006).

[27] Valenzuela, S. O. \& Tinkham, M. Electrical detection of spin currents: The spin-current 
induced Hall effect. J. App. Phys 101, $09 \mathrm{~B} 103$ (2007).

[28] Kimura, T., Otani, Y., Sato, T., Takahashi, S. \& Maekawa, S. Room-Temperature Reversible Spin Hall Effect. Phys. Rev. Lett. 98, 156601 (2007).

[29] Savero Torres, W., Sierra, J.F., Benítez, L.A., Bonell, F., Costache, M.V. \& Valenzuela, S.O. Spin precession and spin Hall effect in monolayer graphene/Pt nanostructures. 2D Mater. 4, 041008 (2017).

[30] Raes, B., Scheerder, J. E., Costache, M. V., Bonell, F., Sierra, J. F., Cuppens, J., Van de Vondel, J., \& Valenzuela S. O. Determination of the spin-lifetime anisotropy in graphene using oblique spin precession. Nat. Commun. 7, 11444 (2016).

[31] Lesne, E., Fu, Y., Rojas-Sánchez, J. C., Vaz, D.C., Naganuma, H., Sicoli, G., Attané, J. -P, Jamet, M., Jacquet, E., George, J. -M, Barthélémy, A., Jaffrès, H., Fert, A., Bibes, M. \& Vila, L. Highly efficient and tunable spin-to-charge conversion through Rashba coupling at oxide interfaces. Nat. Mater. 15, 1261 (2016).

[32] Ferreira, A., Rappoport, T. G., Cazalilla, M. A. \& Castro Neto, A. H. Extrinsic Spin Hall Effect Induced by Resonant Skew Scattering in Graphene Phys. Rev. Lett. 112, 066601 (2014).

[33] Ghiasi, T.S., Kaverzin, A. A., Blah, P. J., \& van Wees, B. J. Charge-to-spin conversion by the Rashba-Edelstein effect in 2D van der Waals heterostructures up to room temperature.

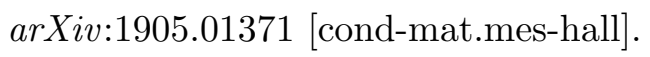

[34] Gebeyehu, Z. M., Parui, S., Sierra, J. F., Timmermans, M., Esplandiu, M. J., Brems, S., Huyghebaert, C., Garello, K., Costache, M. V., \& Valenzuela, S. O. Spin communication over $30 \mu \mathrm{m}$ long channels of chemical vapor deposited graphene on $\mathrm{SiO}_{2}$ 2D Mater. 6, 034003 (2019).

Acknowledgments We thank D. Torres for help in designing Fig. 1. This research was partially supported by the European Union's Horizon 2020 research and innovation programme Graphene Flagship CORE 2, under grant agreement No. 785219, by the European Research Council under Grant Agreement No. 306652 SPINBOUND, by the Spanish Ministry of Economy and Competitiveness, MINECO (under Contracts FIS2015-62641-ERC, MAT2016-75952-R, and SEV-2017-0706 Severo Ochoa), and by the CERCA Programme and the Secretariat for Universities and Research, Knowledge Department of the Generalitat de Catalunya 2017 SGR 827. M.T. acknowledges support from the European Unions Horizon 2020 research and innovation programme under the Marie 
Sklodoswa-Curie grant agreement N 665919.

Author contributions L.A.B. and S.O.V. designed the device and L.A.B. and J.F.S. fabricated it. The measurements were performed by L.A.B. and W.S.T. with the participation of J.F.S. The experimental set-up was implemented by M.T. and M.V.C., who also helped with the measurements. J.H.G. and S.R. carried out the SHE quantum simulations. L.A.B., W.S.T. and S.O.V analyzed the data and wrote the manuscript. All authors contributed to the study, discussed the results and commented on the manuscript. S.O.V. supervised the work. 

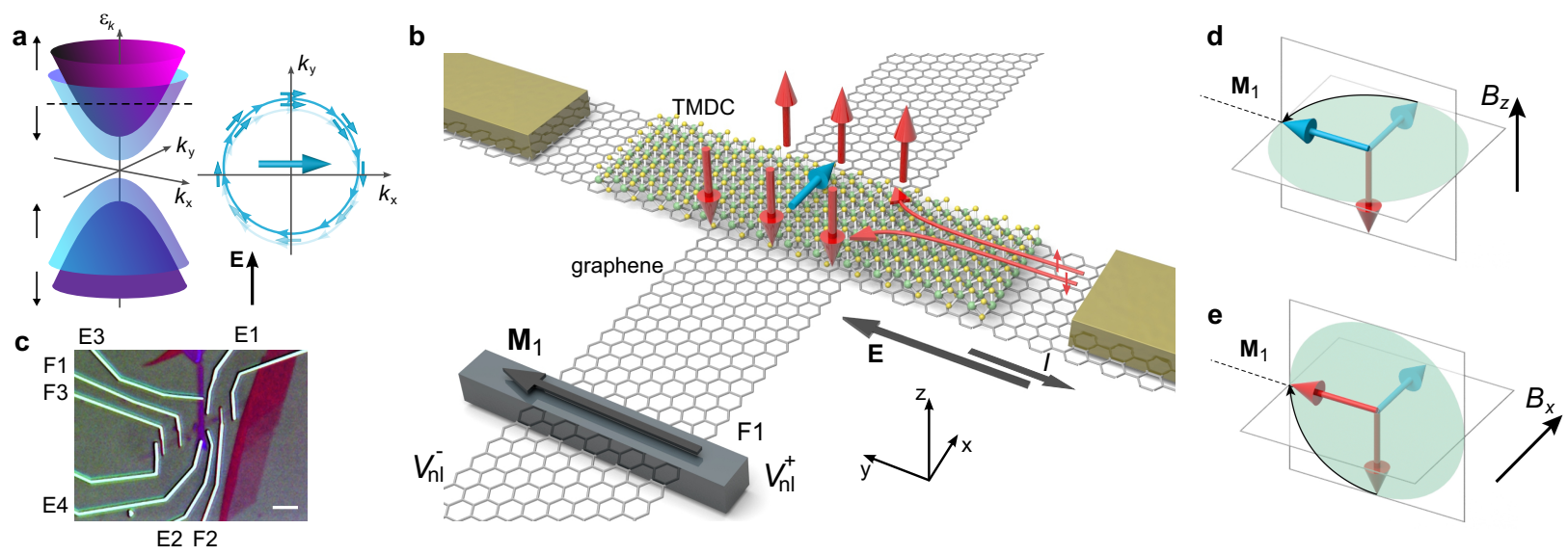

FIG. 1. Spin-to-charge conversion in graphene by proximity of a TMDC and measurement scheme. a, Left: Spin-split bands with opposite spin helicity for graphene-TMDC (blue and purple). The black arrows represent the out-of-plane spin component. Right: Illustration of a microscopic picture of the ISGE. Spin texture for one of the bands developing a non-equilibrium spin density (blue arrow) in an applied electric field $E$. The opposite helical spin texture with lower Fermi wave vector, and smaller contribution to the spin density, is not drawn for clarity. b, Schematics illustrating the basic concepts of the measurement approach. The main elements of the device include a graphene Hall cross with a TMDC strip over one of the arms and a ferromagnet (F1) contacting the other. A current $I$ generated by an electric field $E$ in $\hat{\mathbf{y}}$, along graphene-TMDC, induces a non-equilibrium spin density due to the ISGE with spins along $\hat{\mathbf{x}}$ (blue arrow, see a). Spin accumulation with spins out of plane (along $\hat{\mathbf{z}}$ ) are generated by the SHE with opposite orientation at opposite edges of the graphene-TMDC (red arrows). The induced spins diffuse in graphene towards $\mathrm{F} 1$ and are detected by measuring $V_{\mathrm{nl}}^{\mathrm{F}}=V_{\mathrm{nl}}^{+}-V_{\mathrm{nl}}^{-}$. At zero magnetic field $V_{\mathrm{nl}}^{\mathrm{F}}=0$, as the ISGE and SHE spins are perpendicular to the F1 magnetization $\mathbf{M}_{1}$. $\mathbf{c}$, Optical image of a device with TMDC $=\mathrm{WS}_{2}$. Besides F1 and the Hall cross, the device comprises additional ferromagnetic electrodes $(\mathrm{F} 2, \mathrm{~F} 3)$ and contacts to graphene (E1, E2) and the $\mathrm{WS}_{2}(\mathrm{E} 3, \mathrm{E} 4)$. d, ISGE detection. A magnetic field $B_{z}$ induces spin precession on the ISGE generated spins (blue arrow), leading to a component along $\mathbf{M}_{1}$ and $V_{\mathrm{nl}} \neq 0$. The SHE spins (red arrow) do not contribute to $V_{\mathrm{nl}}$ as they are parallel to $B_{z}$ and do not precess. e, SHE detection. Similar to d, a magnetic field $B_{x}$ induces spin precession on the SHE generated spins leading to $V_{\mathrm{nl}} \neq 0$. The ISGE spins do not precess and do not contribute to $V_{\mathrm{nl}}$. 

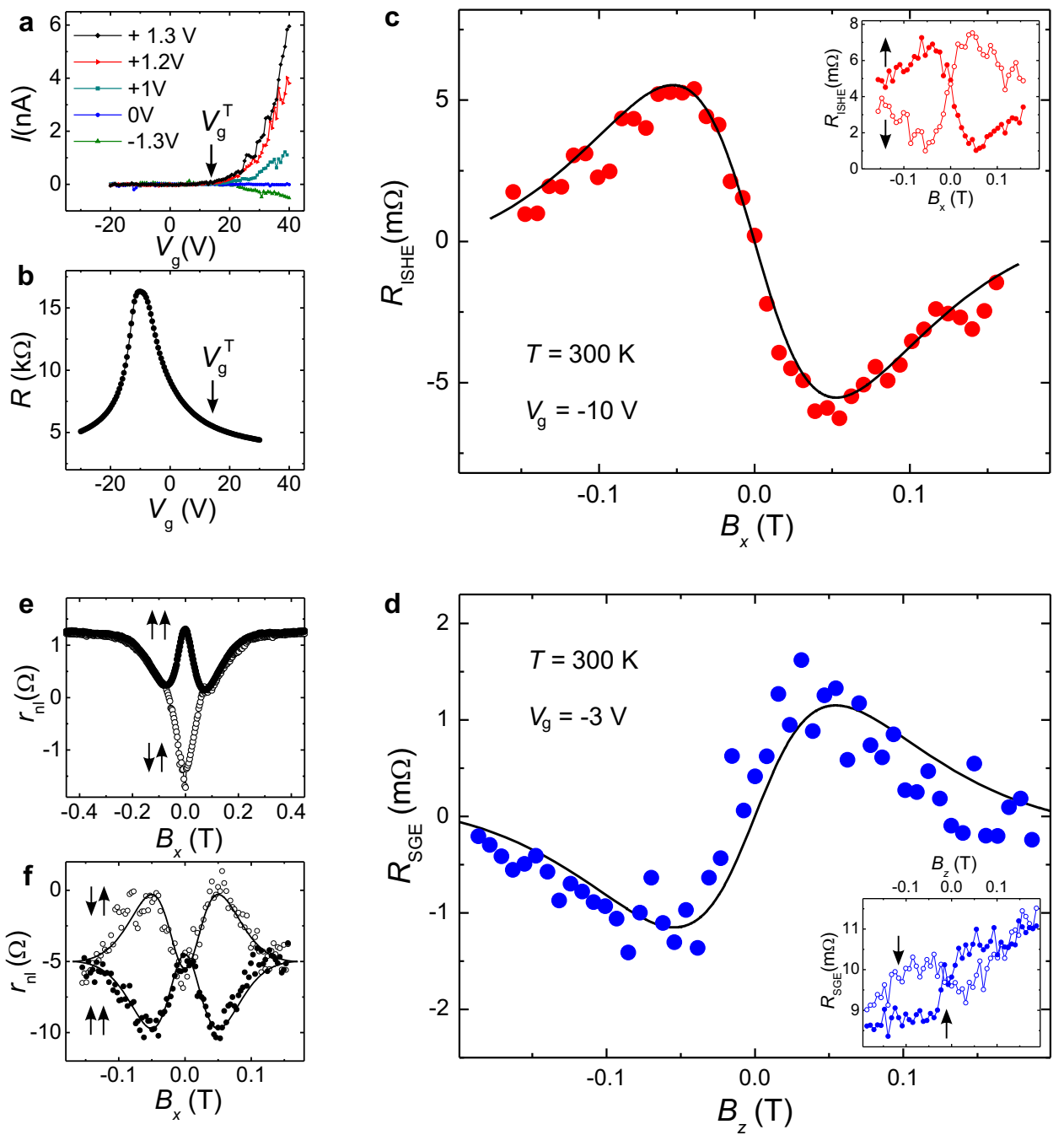

FIG. 2. Sample characterization and spin-to-charge conversion measurements. a, Transfer characteristics $I_{\mathrm{ds}}$ versus $V_{\mathrm{g}}$ for different bias voltage $V_{\mathrm{ds}}$ in graphene- $\mathrm{WS}_{2} ; V_{\mathrm{g}}^{\mathrm{T}}$ indicates the back-gate voltage at which $I_{\mathrm{ds}}$ is first observed. b, Resistance $R$ vs. $V_{\mathrm{g}}$ along graphene- $\mathrm{WS}_{2}$. The charge neutrality point is located at $V_{\mathrm{g}} \approx-10 \mathrm{~V}$, well below $V_{\mathrm{g}}^{\mathrm{T}}$. c, Representative roomtemperature non-local measurements in the ISHE configuration. Inset: $R_{\mathrm{nl}}^{\downarrow, \uparrow}$ vs. $B_{x}$ for $\mathbf{M}_{1}$ antiparallel ( $\downarrow$, open symbols) and parallel ( $\uparrow$, solid symbols) to $\hat{\mathbf{y}}$. Main panel: $R_{\mathrm{ISHE}}=\left(R_{\mathrm{nl}}^{\uparrow}-R_{\mathrm{nl}}^{\downarrow}\right)$. d, As in $\mathbf{c}$ in the SGE measurement configuration. e, Spin injection and detection using F1 and F3 vs $B_{x}$ (see Fig. 1c and Supplementary Fig. 1) for antiparallel (open symbols) and parallel (solid symbols) configuration of the electrodes magnetizations. f, As in e using F1 and F2. The signal becomes larger when $B_{x} \neq 0$ than at $B_{x}=0$, indicating a large spin relaxation anisotropy in graphene- $\mathrm{WS}_{2}$ with long spin lifetimes out of the graphene plane. Solid lines in $\mathbf{c}, \mathbf{d}$ and $\mathbf{f}$ are fittings to the solution of the anisotropic spin diffusion equations. Measurements in $\mathbf{a}, \mathbf{b}, \mathbf{e}$ and $\mathbf{f}$ are performed at $200 \mathrm{~K}$. 

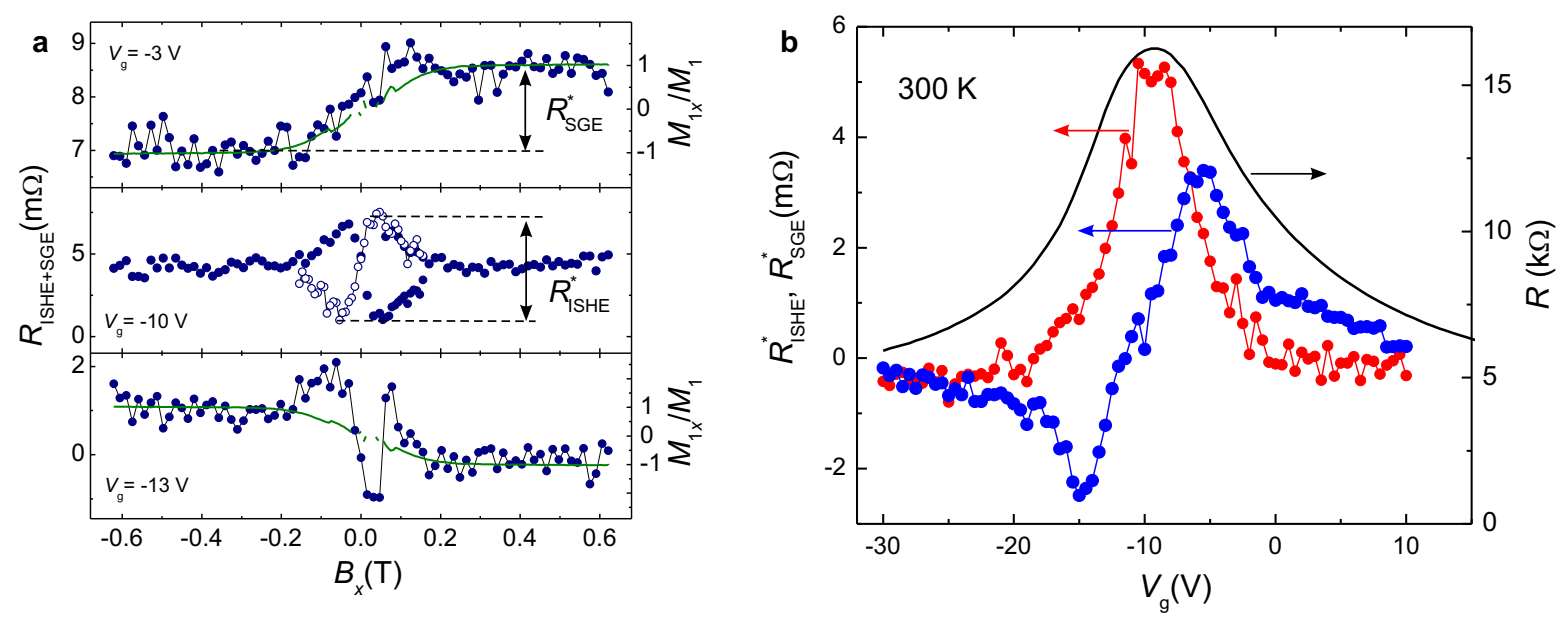

FIG. 3. Gate control of the inverse spin Hall and spin galvanic effects at room temperature. a, Nonlocal resistance $R_{\mathrm{nl}}$ vs $B_{x}$ at selected $V_{\mathrm{g}}$ showing the ISHE precession response at low magnetic fields and the step feature associated to the SGE. The latter is observed when the magnetic field is large enough to rotate $\mathbf{M}_{1}$ and generate a component along $\hat{\mathbf{x}}, M_{1}^{x}$. The normalized $M_{1}^{x}$ (sine of the rotation angle) is extracted from spin precession measurements as in Fig. 2e [26] and represented with green lines. b, Spin-to-charge conversion for the ISHE (red) and the SGE (blue) as a function of $V_{\mathrm{g}}$. The corresponding conversion efficiencies are quantified with $R_{\mathrm{ISHE}}^{*}$ and $R_{\mathrm{SGE}}^{*}$ as defined in a. $R$ vs. $V_{\mathrm{g}}$ is shown for comparison (black line). 


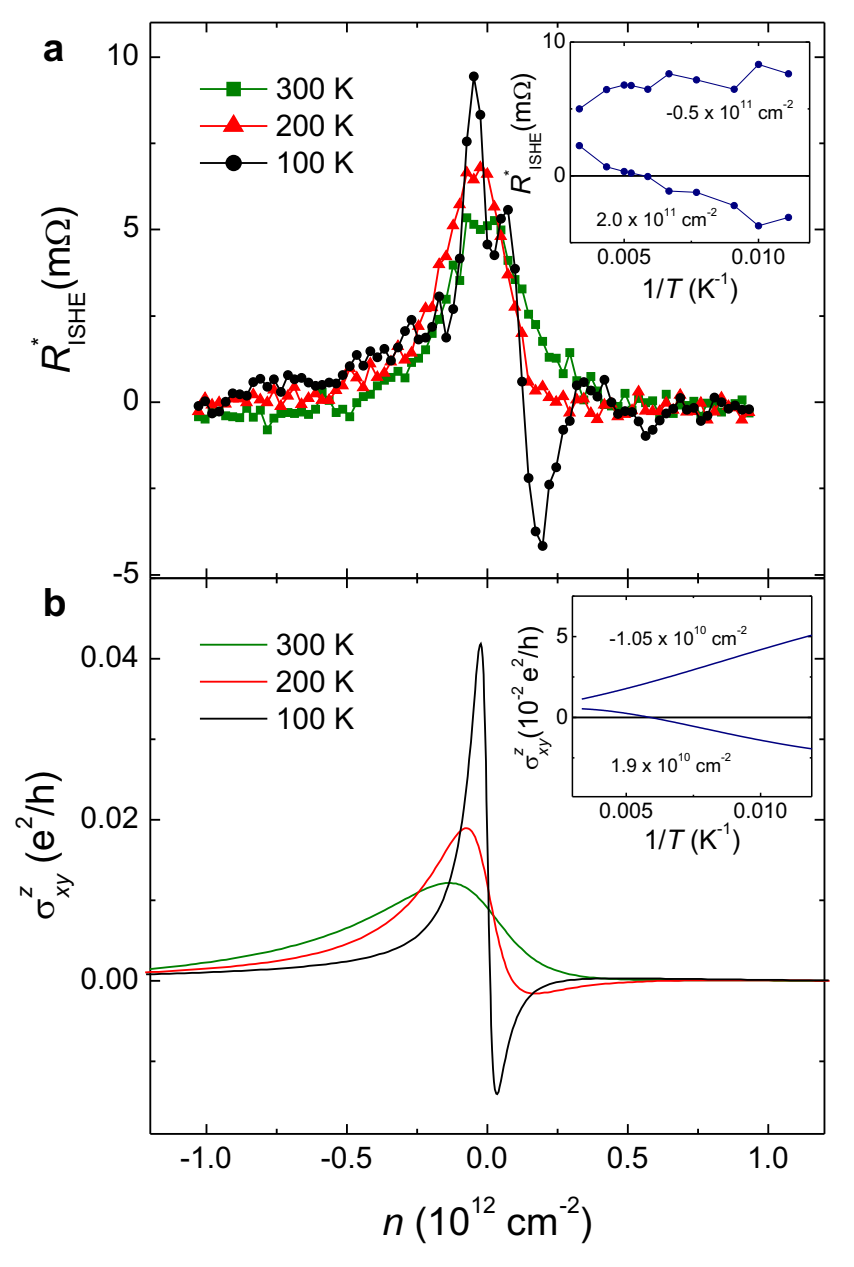

FIG. 4. Temperature dependence of the SHE spin-to-charge conversion. a, $R_{\mathrm{ISHE}}^{*}$ vs. carrier density $n$ at the specified temperatures. b, spin Hall conductivity $\sigma_{x y}^{z}$ in the weak disorder limit using realistic parameters. The insets in $\mathbf{a}$ and $\mathbf{b}$ show the temperature dependence of $R_{\mathrm{ISHE}}^{*}$ (a) and $\sigma_{x y}^{z}$ (b) for $n$ at the extrema positions. 